\title{
Using a Cultural Intelligence Assessment to Teach Global Leadership
}

\author{
Brett L. Whitaker, Ph.D. \\ Assistant Professor of Leadership Studies \\ Fort Hays State University \\ Justin P. Greenleaf, Ph.D. \\ Assistant Professor of Leadership Studies \\ Fort Hays State University
}

\begin{abstract}
This paper describes the practice of students enrolled in an undergraduate Global Leadership course taking a Cultural Intelligence (CQ) assessment. The results of the assessment provided insight into the students' development across various dimensions of CQ, as well as their scores relative to their peers and global averages. This information was incorporated into the course instruction to assist students in developing a more concrete understanding of their own capacities in otherwise ambiguous or abstract behavioral dimensions. Results of the practice indicate that it is beneficial to the students, particularly from a self-efficacy and motivational standpoint. A sample lesson plan and debriefing questions are provided to assist educators desiring to incorporate a CQ assessment into their teaching.

\section{Introduction}

Global leadership, leadership in cross-cultural environments, and comparative leadership are all areas of increasing importance in an ever more connected world (Osland, Bird, \& Oddou, 2012). Addressing learning outcomes related to these areas can sometimes be challenging, particularly for educators that are working with younger students that may have very limited life experience with these types of environments (Caligiuri, 2006). One way to assist students with developing a more thorough and grounded understanding of their own capacity is through the use of a Cultural Intelligence (CQ) assessment that measures students' readiness in four dimensions of CQ. This paper briefly describes the utilization of one such assessment in an undergraduate Global Leadership course. A sample lesson plan is presented, and strategies for debriefing and reflection are discussed.
\end{abstract}

\section{Review of Related Scholarship}

A number of scholarly streams of research support the practice described in this paper. The most obvious is the construct of CQ itself. CQ is one of what scholars have referred to as "multiple intelligences" (Goleman, 1995). Intelligence research began with cognitive intelligence (IQ) in the field of psychology (Yukl, 2010). Many leadership studies scholars are also likely familiar with Emotional Intelligence (EQ) which was described by Goleman and others beginning in the 90's (Goleman, 1995). The central difference between EQ and IQ was 
that EQ was conceptualized as a developmental capacity that could be learned and practiced, whereas IQ was a fixed trait that was relatively static (Early \& Mosakowski, 2004).

As noted by Bird and Stevens (Mendenhall, et al., 2013), the CQ construct is "predicated on the broader conceptual notion of multiple intelligences" (p. 124). CQ follows this development of EQ by also being a developmental set of capacities, but rather than being focused on emotions, it is concerning one's capacity for engaging in cross-cultural scenarios (Earley, Ang, \& Tan, 2006). CQ is defined by Livermore (2011) as, "the capability to function effectively in a variety of cultural contexts" ( p. 3). CQ is conceived of as being a state-like quality, and it is malleable over time. Most researchers refer to a four-dimensional construct of CQ consisting of motivation, sense-making, behavioral skills, and knowledge. The main capacities are composed of multiple sub-dimensions (Van Dyne et al., 2012).

Research on the CQ construct supports the notion that individuals can exhibit different levels of competency on different dimensions independent of one another (Early, Ang, \& Tan, 2006). Livermore (2011) and others have written extensively about the application of these dimensions in cross-cultural situations, but to assist the reader, the following examples are provided to clarify the construct. These dimensions were first described by Early and Ang (Early, Ang, \& Tan, 2006) in their initial research into and development of the CQ construct.

The first dimension of CQ is motivation, also referred to as drive. This dimension describes the level of motivation an individual feels for engaging in cross-cultural interaction. An example is a person's willingness to travel internationally or to take on a new work assignment that involves interaction with diverse stakeholders.

The second dimension of CQ is knowledge, also described as cognition. This dimension is simply the depth and breadth of knowledge that a person possesses about a culture that is different from their own. Examples of cultural knowledge are understanding a foreign language, or understanding spatial differences in verbal communication in different cultures.

Sense-making, also known as meta-cognition or strategy, is the third dimension of CQ. This dimension describes the capability of one to make sense of the world around oneself and the experiences that one is having. This dimension describes the capacity of the individual to properly order and assign correct meaning to inputs that the individual is experiencing. An example of this would be experiencing a person from another culture standing very close during a conversation, and then making sense of the situation by recognizing that spatial differences are culturally contingent.

The final dimension of CQ is action or the behavior component. This dimension describes the readiness that a person has for engaging in the behavior of cross-cultural interaction. To extend the example from above, in the situation described, a person exhibiting high CQ behavior would be able to carry on the conversation at close proximity without undue stress. That person would be exhibiting a high degree of CQ, by being motivated to do a good job in the cross-cultural environment, having knowledge of cultural norms related to personal space, making sense of the situation in the moment, and then behaving in a way that was culturally appropriate. 
CQ is also generally supported in global leadership literature. According to Black and Gregersen (2000), the primary objective of global leadership training is, "stretching someone's mind past narrow domestic borders and creating a mental map of the entire world" (p.175). This objective has often been manifested in the regular use of the CQ construct and CQ assessments in studies of expatriates. One early example of this stream of scholarship was the work of Templer (2006) and his colleagues who found that CQ drive was predictive of successful adjustment of expatriates working in Singapore. Other work on expatriate selection, adjustment, work performance, and leadership behavior suggests CQ may be a good predictor of success in complex, cross-cultural leadership situations (Ang, et al., 2004). One notable exception is the work of Ward and colleagues (2009), who studied international students and found a high degree of convergence with EQ, which brings into question the validity of the severability of the two constructs.

To understand the relationship between CQ and Global Leadership, it's important to note here what definition the authors are using to define the concept of Global Leadership. Global Leadership, like domestic leadership before it, has been defined in many ways and does not have a single or universal definition to which all scholars subscribe. For the purpose of this paper, the authors are referring to Global Leadership as defined by Reiche and Mendenhall (Mendenhall et al., 2013). This definition consists of two construct elements that define the environment in which the Global Leadership is taking place. The first of these is an environment that exhibits a high degree of complexity (e.g. multiplicity, interdependence, ambiguity, flux), and the second is boundary spanning.

Another area of scholarship that informs the current practice is the research related to confidence and academic performance or learning. As described below, one of the main objectives of incorporating this CQ assessment into the classroom was to build the confidence of students who might otherwise struggle to assess their own competencies in cross-cultural environments. As is well established in the literature, learner confidence, sometimes referred to as academic self-efficacy, is positively correlated with a number of academic performance indicators (Tuckman \& Sexton, 1992). Students who can envision themselves engaged in the type of behavior being discussed tend to have greater understanding and higher retention compared with those students who only grasp the content in an abstract fashion (Vrugt, Langereis, \& Hoogstraten, 1997). For this reason, giving students an opportunity to assess their own CQ capacities should increase the opportunity for learning over simply discussing the content with no opportunity for concrete self-assessment. As Scott and Weeks (2015) indicate, this perspective transfers to leadership education and pedagogy well, saying that, "selfexploration, understanding one's true self, and recognizing one's values are all components of authentic leadership development" (p. 140).

Finally, the role of students' self-assessment has been long established as a beneficial pedagogical technique (Sadler, 1989). In addition to providing students with information about their current state of development in a given academic area, self-assessment is also beneficial in helping students to envision their own growth and development. As Black and Wiliam (1998) indicate, "where anyone is trying to learn, feedback about their efforts has three elements - the desired goal, the evidence about their present positon, and some understanding of a way to close the gap between the two" (p. 136). The first of these items can and should be provided by the 
instructor, but self-assessment can be very useful in both the second and third components of this process. By allowing students to self-assess their CQ, they gain both understanding and motivation to improve in their areas of weakness and augment their strengths.

\section{Description of the Practice}

The CQ assessment used in this particular course is a proprietary instrument developed and published by the Cultural Intelligence Center (CIC) (disclosure note: the authors do not represent CIC and have no relationship with them other than having purchased their assessment in past semesters for utilization in a course). Utilization of this particular instrument was beneficial in a number of ways for use in an academic class. First, the instrument has been validated and found to be a reliable measure of CQ dimensions. Second, by contracting with a vendor, the workload for the instructor is reduced in coding, analysis and scoring of the instrument. Finally, the most important benefit is that, by having a custom instrument generated for each course, the instructor and students are provided valuable feedback on their results relative to others. More detail will be provided on this topic in the discussion of the outcomes.

Gaining access to the instrument was fairly straightforward. Prior to the beginning of the semester, the instructor made arrangements with representatives of the CIC for the custom instrument. The need to purchase access to the survey was noted as part of the books and course materials section of the syllabus, and students were provided advanced notification of this on the first day of class. Students paid approximately $\$ 18$ each in order to purchase access. Various options (pre-post, group report, etc.) alter the cost. Further information about the various products available can be accessed directly from the vendor at http://www.culturalq.com/tmpl/assessments/assessments.php.

The application of this CQ assessment took place in an undergraduate Global Leadership course at a public, Midwestern university. The course in question is an upper division course offered through the Leadership Studies department and consists of approximately 25 students taught in a traditional face-to-face format. Students enrolled in the class are generally either Leadership Studies majors or are seeking a Global Leadership Certificate, so all students coming into the class have previous exposure and experience with western leadership theory. Students are generally traditional aged, primarily Caucasian, of varied economic backgrounds, and usually from rural home environments. One of the most common characteristics of the students enrolled in the class is their limited exposure to diverse cultures, and little or no international travel experience.

In the Global Leadership courses in which this instrument has been utilized, students were first introduced to the concept of CQ and the foundational scholarship supporting this area. Following this, and approximately half way through the academic term, the students were provided instructions on how to access and complete the CQ assessment. The CIC provided a simple URL link for students to follow and enter their access code. The instrument was completed outside of class time and took students an average of about 20 minutes to complete. The instructor is provided a copy of the results within about one week of the closing of the survey session. 
Concurrently with students taking the test outside of class, they are also tasked with doing background reading about CQ on their own, and the materials covered are addressed in class periods leading up to the sessions discussing their assessment results. During the first class period that is dedicated to discussing the results, the instructor begins by distributing to each student his or her individual assessment results. The materials that each student receives are listed below.

1. A brief overview of CQ and the components of the assessment.

2. Scoring on each of the four dimensions of CQ measured by the assessment (Drive, Knowledge, Strategy, and Action).

3. Detailed scoring on sub-dimensions within each of the four dimensions.

4. Comparative data showing their scores relative to their peers and worldwide averages.

5. A customized CQ development plan and reflective questions and prompts to help students think through interpretation of their scores and areas in which they may be able to improve.

Following the distribution of these individual reports, the instructor leads the students through a discussion and debrief about their results. One useful strategy in this debrief session is to place scoring numbers along a wall in the classroom, and to have students physically organize themselves according to their score on various dimensions. Students often respond that they have a better understanding of their capacities relative to their peers after seeing the physical manifestation of their scores by placement.

Faculty may choose to engage with the students using the assessment data in a number of ways. A sample lesson plan of one potential way to facilitate this conversation in an hour-long class period could look like this:

1. Distribute report packets, and give students time to read the basic information about their scores on each dimension. (10 minutes)

2. Facilitate discussion about each dimension, reminding students about what the dimension was assessing, and having them order themselves along the wall according to their scores. The students should be rearranged for each dimension as it is discussed. This allows them to have a visual and interactive interpretation of their results relative to their peers. The instructor may elect to stand in a place that represents a national average for the particular dimension being discussed. (30 minutes)

3. Engage students in reflective conversation about their relative strengths and weaknesses. The instructor may utilize some of the questions listed below, or may allow for more open-ended discussion prompted by the students. (10 minutes)

4. Have students write out some basic strategies for their own CQ development and share with a peer or with the whole class. (10 minutes)

A central component of successfully facilitating the CQ assessment is asking quality debriefing questions. The particular set of questions and process of facilitation should be developed by the instructor to accommodate the particular circumstances of the course and students. Some general questions are shared below as examples of the types of questions that might be asked of students and are organized by lower and higher order thinking. 
Lower order questions:

1. In which sub-dimension of CQ did you score the lowest? The highest?

2. What are your results relative to your peers or national averages?

3. What do your score results mean for your behavior when going into a new environment?

4. How is CQ connected to leadership and the process of creating positive organizational and community change?

Higher order questions:

1. What life experiences have caused you to develop the capacities that are indicated on your CQ assessment?

2. How might you utilize this feedback in your career?

3. What strategies could you employ to address your weakest sub-dimension of CQ?

4. Why or why not do your CQ assessment results give you confidence?

5. What implications do the results have for your ability to engage in leadership processes from a cross-cultural perspective?

6. When collaborating with others to create change, how would a cultural scenario that demonstrated your strengths be designed? How would one that exposed your weaknesses be designed?

\section{Discussion of Outcomes/Results}

This assessment instrument and report have been utilized several times in a Global Leadership course with undergraduate students. Overall outcomes of the program have been highly favorable from student feedback. In particular, the assessment seems to address one of the most challenging elements of teaching cross-cultural or global leadership: the ability for students to feel meaningfully engaged with diverse perspectives.

Often, students enrolled in the classes in the program are local, domestic students that lack extensive experience in global or cross-cultural environments. As a result, it is difficult for these students to picture themselves in these types of challenging multicultural circumstances, and even more challenging for them to assess their readiness to engage in these environments. By providing students with a concrete and quantifiable measure of their capacity in $\mathrm{CQ}$, this instrument allows for a deeper and more purposeful conversation in the classroom related to student development in these dimensions. Students often express that they feel added confidence moving forward having completed the assessment and gained insight into their own strengths and weaknesses. For example, one student was quoted as saying, "Taking this CQ test showed me my strengths and weaknesses so now I'm aware of where I stand, but also informed on where I need to be." A different student commented, "I found the CQ assessment to be very humbling, but also it gave me confidence about my own abilities."

Feedback from students who have taken the CQ assessment is positive. One student who recently completed the self-assessment said, in regards to her own learning, "I really liked learning about my CQ assessment because it was a tangible thing that I can take, look at, and improve in my own capacities." Another student indicated that, "I have always had the desire to travel the world, but lacked the drive out of fear of the unknown. Learning about CQ stretched me in ways that I never thought possible." Many other students have shared similar reactions to 
the content of CQ and the insights gained through the self-assessment. For example, in reflecting on the future, one student commented, "Knowing my personal CQ capabilities will be helpful to me in my career because I know where my abilities currently rank, and I know in what areas I am lacking."

Several weaknesses of the assessment should also be acknowledged. In addition to the monetary expense for students, there is an expense of class time and attention needed to adequately debrief students after they access their results. It would be ill-advised to simply have students complete the assessment as an independent activity and never debrief their results as a group. Students on occasion will score low in a particular dimension, and while this is valuable information for them to have developmentally, it can be discouraging or frustrating to them if they are not adequately debriefed. Successfully navigating and reflecting on this information can be a catalyst to encourage student development, but neglecting this critical step can severely limit the utility of the assessment.

\section{Reflections of the Practitioner}

It has been helpful that most of the students in the course have some shared experience in leadership studies and background in domestic leadership theory. This foundational understanding of leadership theory has proven useful in debriefing the assessment as the students try to make connections between CQ and leadership theory and practice. While the topic of CQ is discussed generally in some of these previous classes, it is beneficial for the students to have additional exposure to the topic that is specifically focused on CQ. In relating to the course content, one student commented, "When I think critically about what I have learned in this class, the specific topic that I can most readily utilize in my life is CQ knowledge and CQ strategy."

In reflecting on the students, many are from similar backgrounds. The students at the university primarily come from small, rural environments which have little cultural diversity, and most of the students have little to no international travel experience. While this activity certainly does not take the place of an authentic cross-cultural experience (e.g. a study abroad experience), it can serve as a catalyst for getting students to think about cultural differences and feel more comfortable engaging in different environments. For example, one student commented, "I am far more confident now going into cross-cultural situations after learning about my own CQ capabilities."

With respect to the facilitation of the discussion/debrief, the most common challenge is helping students make meaningful connections between the CQ assessment and their personal lives. Often students will make benign interpretations of the results, perhaps out of a lack of critical thinking or a desire to protect self. In instances like this, the challenge of the practitioner is to guide the student to consider possible alternative interpretations without pushing the students further than they are comfortable. While this might be uncomfortable for some students and/or instructors, the learning that takes place is worth the effort. As one student stated, "My understanding of cultural intelligence will be highly beneficial in my life, no matter what organization I am a part of." 
From the perspective of an educator/practitioner, this assessment and the results that are presented to students are highly valuable. The authors of this paper teach at a Midwest public institution and often encounter students that are lacking confidence and experience in assessing their readiness to engage in cross-cultural environments. This assessment has been a valuable addition to enhance the pedagogy of the course. In particular, utilizing this assessment has brought a sense of concreteness and reality to topics that can otherwise seem very abstract or removed for students.

\section{Recommendations}

The CQ assessment discussed in this paper is recommended for use in courses that address cross-cultural or global leadership, intercultural communication, comparative leadership, or in other educational settings that incorporate these or related learning outcomes. It can be particularly helpful when engaging a group of students that have little background in crosscultural environments or experience working in diverse settings.

It is recommended that, prior to the administration of the CQ assessment, the practitioner explain the purpose and intent of the assessment. Helping the students understand that the assessment merely describes their default tendencies rather than define who they are is an important first step in having meaningful conversations. Equally important to this conversation is helping the students recognize that the four dimensions are able to be developed and, with time and practice, weaknesses can be addressed and strengths can be enhanced.

Finally, while the CQ assessment has not been used for purposes of assessment and evaluation at the author's institution, it certainly could be used towards that end for institutions that desire to measure progress in this area. For future practitioners interested in using the CQ assessment in classes that have a heavy focus on cultural intelligence, it may be useful to utilize the CQ assessment in the form of a pre-post test. This would have particular relevance to courses that have identified outcomes that align with one or more of the CQ dimensions. 


\section{References}

Black, P. \& Wiliam, D. (1998). Inside the black box: Raising standards through classroom assessment. Phi Delta Kappan, 80(2), 139-148.

Black, J. S. \& Gregersen, H. B. (2000). High impact training: Forging leaders for the global frontier. Human Resource Management, 39(2), 173-184.

Caligiuri, P. M. (2006). Developing global leaders. Human Resource Management Review, 16, $219-228$

Earley, P. C., Ang, S., \& Tan, J. (2006). CQ: Developing cultural intelligence at work. Stanford, CA: Stanford University Press.

Earley, P. C. \& Mosakowski, E. (2004). Cultural intelligence. Watertown, MA: Harvard Business Review.

Goleman, D. (1995). Emotional intelligence. New York: Bantam Books, Inc.

Livermore, D. (2011). The cultural intelligence difference. New York: AMACOM.

Mendenhall, M. E., Osland, J. S., Bird, A., Oddou, G. R., Maznevski, M. L., Stevens, M. J., \& Stahl, G. K. (2013). Global leadership: Research, practice, and development. New York: Routledge.

Osland, J. S., Bird, A., \& Oddou, G. (2012). The context of expert global leadership. In W.H.

Mobley, Y. Wang, and M. Li (eds) Advances in Global Leadership, vol. 7. Oxford: Elsevier.

Sadler, R. (1989). Formative assessment and the design of instructional systems. Instructional Science, 18, 119-144.

Scott, M. \& Weeks, P. P. (2015). Using film to teach authentic leadership. Journal of Leadership Education, 15(1), 140-149.

Templer, K., Tay, C., \& Chandrashekar, N. A. (2006). Motivational cultural intelligence, realistic job preview, realistic living conditions preview and cross-cultural management. Group and Organizational Management, (3), 154-171.

Tuckman, B., \& Sexton, T. L. (1992). The effects of informational feedback and self-beliefs on the motivation to perform a self-regulated task. Journal of Research in Personality, 26, $121-127$. 
Van Dyne, L., Ang, S., Ng, K. Y., Rockstuhl, T., Tan, M. L., \& Koh, C. (2012). Sub-dimensions of the four factor model of cultural intelligence: Expanding the conceptualization and measurement of cultural intelligence. Social and Personal Psychology: Compass, 6(4), 295-313.

Vrugt, A. J., Langereis, M. P., \& Hoogstraten, J. (1997). Academic self-efficacy and malleability of relevant capabilities as predictors of exam performance. Journal of Experimental Education, 66(1), 61-72.

Ward, C., Fischer, R., Lam, F. S. Z., \& Hall, L. (2009). The convergent, discriminant, and incremental validity of scores on a self-report measure of cultural intelligence. Education and Psychological Measurement, 69(1), 85-105.

Yukl, G. (2010). Leadership in organizations (7 ed.). Upper Saddle River, NJ: Prentice Hall.

\section{Author Biographies}

Brett L. Whitaker is an Assisstant Professor in the Department of Leadership Studies at Fort Hays State University. Dr. Whitaker develops curriculum and conducts research focused on global leadership and leadership in cross-cultural environments, and serves as an administor for international leadership studies initiatives. He may be reached at blwhitaker@fhsu.edu.

Justin P. Greenleaf is an Assistant Professor in the Department of Leadership Studies at Fort Hays State University. Dr. Greenleaf focuses his research on the practical application of leadership theory and practice. He teaches primarially at the undergraduate level. He may be reached at jpgreenleaf@fhsu.edu. 